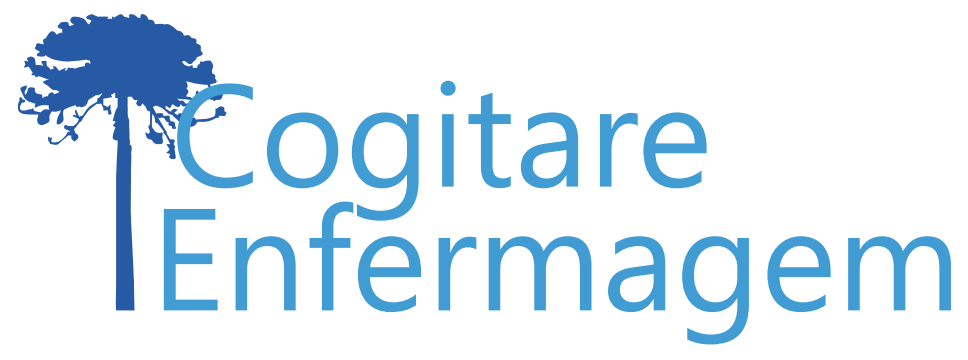

\title{
VALIDAÇÃO DE PROGRAMA ELETRÔNICO PARA SEGUIMENTO DO RECÉM-NASCIDO COM SÍFILIS CONGÊNITA
}

Samara Fernanda Vieira Valença ${ }^{1}$, Camila Aparecida Pinheiro Landim Almeida², Jaqueline Carvalho Silva e Sales ${ }^{3}$, Carmen Viana Ramos ${ }^{4}$, Luana Kelle Batista Moura ${ }^{5}$, Ellen Thallita Hill Araújo ${ }^{6}$

\section{RESUMO}

Objetivo: validar o conteúdo e a aparência de um programa eletrônico para seguimento clínico do recém-nascido com sífilis congênita.

Método: estudo metodológico, de validação de aparência e conteúdo. Os dados foram coletados de junho a agosto de $2018 \mathrm{com}$ instrumentos de avaliação para validação por sete especialistas médicos docentes de uma Instituição de Ensino Superior, com experiência em pediatria e/ou infectologia, com tempo de formação superior a 16 anos. Na análise dos dados, utilizou-se o Índice de Validade de Conteúdo com valor estabelecido de 0,78.

Resultados: quanto à estrutura e apresentação, objetivos e relevância, todos os itens foram considerados validados pelos especialistas. O Indice de Validade de Conteúdo global foi 0,97.

Conclusão: o programa eletrônico foi validado quanto ao conteúdo e aparência, tendo como contribuição, aos enfermeiros e médicos da Atenção Básica, a produção de uma ferramenta de fácil utilização para o efetivo seguimento clínico ao recém-nascido com sífilis congênita.

DESCRITORES: Estudos de Validação; Tecnologia Educacional; Sífilis Congênita; Recém-nascido; Atenção Primária à Saúde.

\section{COMO REFERENCIAR ESTE ARTIGO:}

Valença SFV, Almeida CAPL, Sales JCS e, Ramos CV, Moura LKB, Araújo ETH. Validação de programa eletrônico para seguimento do recém-nascido com sífilis congênita. Cogitare enferm. [Internet]. 2020 [acesso em "colocar data de acesso, dia, mês abreviado e ano"]; 25. Disponível em: http://dx.doi.org/10.5380/ce.v25i0.62829.

\section{(c) (1)}

Este obra está licenciado com uma Licença Creative Commons Atribuição 4.0 Internacional.

${ }^{1}$ Médica. Mestranda em Saúde da Família. Centro Universitário Uninovafapi. Teresina, PI, Brasil.

${ }^{2}$ Enfermeira. Doutora em Enfermagem. Docente de Enfermagem do Centro Universitário Uninovafapi. Teresina, PI, Brasil. 9

${ }^{3}$ Enfermeira. Doutora em Enfermagem. Docente de Enfermagem da Universidade Federal do Piauí. Teresina, PI, Brasil.

${ }^{4}$ Nutricionista. Doutora em Saúde da Criança e da Mulher. Docente do Centro Universitário Uninovafapi. Teresina, PI, Brasil. $\bigcirc$

${ }^{5}$ Cirurgiã-Dentista. Doutora em Endodontia. Docente do Centro Universitário Uninovafapi. Teresina, PI, Brasil. (?)

${ }^{6}$ Enfermeira. Mestranda em Saúde da Família. Centro Universitário Uninovafapi. Teresina, PI, Brasil. $\left(\frac{)}{0}\right.$ 


\title{
VALIDATION OF AN ELECTRONIC TOOL FOR MONITORING NEWBORNS WITH CONGENITAL SYPHILIS
}

\begin{abstract}
Objective: To validate the content and appearance of an electronic tool for the monitoring of newborns with congenital syphilis.

Method: Methodological study of validation of content and appearance. Data were collected from June to August 2018 with the use of assessment instruments for validation by seven medical specialists who teach at a higher education institution, with experience in pediatrics and/or infectious diseases, and who have completed their undergraduate courses more than 16 years ago. Content Validity Index with an established value of 0.78 was used.

Results: Regarding structure and presentation, objectives and relevance, all items were considered validated by the experts. The global Content Validity Index was 0.97.

Conclusion: The electronic tool was validated for content and appearance, and its contribution to nurses and doctors in primary care was the production of an easy-to-use tool for effective clinical monitoring of infants with congenital syphilis.
\end{abstract}

DESCRIPTORS: Validation Studies; Educational technology; Congenital syphilis; Newborn; Primary Health Care.

\section{VALIDACIÓN DE UN PROGRAMA ELECTRÓNICO PARA EL SEGUIMIENTO DE RECIEN NACIDOS CON SIFILIS CONGENITA}

\begin{abstract}
RESUMEN:
Objetivo: validar el contenido y la apariencia de un programa electrónico para el seguimiento clínico del recién nacido con sífilis congénita.

Método: Estudio metodológico, validación de apariencia y contenido. Los datos fueron recopilados de junio a agosto de 2018 con instrumentos de evaluación para su validación por parte de siete especialistas médicos del cuerpo docente de una institución de enseñanza superior, con experiencia en pediatría y/o infectología, con un período de formación de más de 16 años. Los datos se analizaron utilizando el Índice de Validez de Contenido con un valor establecido de 0,78.

Resultados: en lo que respecta a la estructura y presentación, los objetivos y la relevancia, todos los ítems fueron considerados validados por parte de los especialistas. El Índice de Validez de Contenido global fue de 0,97.

Conclusión: el programa electrónico fue validado en cuanto a su contenido y apariencia, en el cual contribuyeron enfermeros y médicos del área de Atención Básica y la producción de una herramienta de fácil utilización para un efectivo seguimiento clínico del recién nacido con sífilis congénita.
\end{abstract}

DESCRIPTORES: Estudios de validación; tecnología educativa; sífilis congénita; recién nacido; atención primaria de salud. 
A sífilis é uma doença infectocontagiosa de caráter sistêmico, passível de prevenção, que, quando não tratada precocemente, pode evoluir para complicações graves e sequelas irreversíveis. A transmissão pode acontecer por via sexual, sanguínea (transfusão sanguínea) ou mãe-filho (transmissão vertical)(1).

Em meio às doenças que podem ser adquiridas durante o período gravídicopuerperal, a sífilis possui as maiores taxas de transmissão. Ao contrário de muitas infecções neonatais, essa patologia encontra-se inserida no quadro de causa perinatal evitável e pode ser controlada mediante diagnóstico e tratamento efetivo durante a gestação ${ }^{(2-3)}$.

A Sífilis Congênita (SC) é decorrente da disseminação do Treponema pallidum, via transplacentária da gestante não tratada ou tratada inadequadamente para o seu concepto em qualquer momento da gestação. $A$ infecção pode causar consequências graves para o concepto, desde sequelas neurológicas até o óbito fetal ${ }^{(1,4)}$.

No ano de 2016, foram notificados no Brasil cerca de 87.593 casos de sífilis adquirida, destes 37.436 casos de sífilis foram em gestantes, sendo 20.474 casos de SC. No que se refere aos óbitos pela patologia em crianças brasileiras menores de um ano de idade, sobressai a taxa de 18,1 óbitos/1.000 nascidos vivos ${ }^{(5)}$.

A incidência da sífilis congênita é um importante indicador da qualidade da atenção à saúde materno-infantil. A persistência de altas taxas de incidência da doença e da transmissão vertical evidenciam falhas na cobertura e qualidade do pré-natal(6-7).

Dente os principais fatores responsáveis pelos elevados índices da sífilis congênita em todo país, destacam-se: sorologia para sífilis não realizada nos períodos preconizados; interpretação inadequada da sorologia; falha no reconhecimento dos sintomas; falta de tratamento do parceiro sexual; e falha na comunicação entre os profissionais de saúde ${ }^{(8)}$.

Nesta perspectiva, o Ministério da Saúde lançou o "Plano de Eliminação da Sífilis Congênita", em conformidade com a proposta de controle do agravo nas Américas e Organização Mundial da Saúde, e estabeleceu essa patologia como uma das prioridades para notificação compulsória, implantação de ações de prevenção e controle de infecções sexualmente transmissíveis ${ }^{(5,9)}$.

Embora o diagnóstico e o tratamento sejam de fácil acesso e de baixo custo, há necessidade de implementação das normas de orientação e do rastreamento sistemático por meio de programas eletrônicos que possam melhorar a qualidade da terapêutica da $\mathrm{SC}^{(10)}$.

Desta forma, mediante a necessidade de se garantir cuidados de qualidade, com consequente diminuição dos riscos para o paciente, têm-se a necessidade de uma ferramenta eletrônica validada com as linhas de cuidados para prevenção e seguimento da assistência ao recém-nascido (RN) com sífilis congênita ${ }^{(11,12)}$.

No processo de trabalho dos profissionais de saúde nos serviços de atenção básica, a validação científica para utilização de programas eletrônicos na gestão dos cuidados no pré-natal e acompanhamento da gestante se torna importante para melhoria do atendimento prestado ${ }^{(11-12)}$.

Diante dessa problemática, esse estudo teve como objetivo validar o conteúdo e a aparência de um programa eletrônico para seguimento clínico do recém-nascido com sífilis congênita, a fim de possibilitar a utilização por profissionais de saúde, para promover o seguimento adequado destes pacientes na Atenção Primária à Saúde. 
Trata-se de um estudo metodológico de abordagem descritiva, desenvolvido pelas etapas proposta por Echer: a primeira foi a elaboração da ferramenta; e, a segunda, a validação de conteúdo mediante a avaliação dos instrumentos por juízes/especialistas ${ }^{(13)}$.

Na primeira etapa, o programa foi elaborado fundamentando-se no levantamento teórico sobre o seguimento do recém-nascido com sífilis congênita. Posteriormente, reuniram-se também as informações da Linha de Cuidados para a Atenção Integral recémnascido com sífilis e protocolo aḑotado pelo Ministério da Saúde. Em seguida, a construção do programa eletrônico, validação de conteúdo, aparência e adaptação do material por meio de especialistas peritos ${ }^{\left(5,13^{3}-15\right)}$.

O programa eletrônico foi denominado "Seguimento do recém-nascido com sífilis congênita", cuja população-alvo são os profissionais, médico e enfermeiro, da atenção básica. O programa elaborado é um prontuário eletrônico, o qual contemplou o fluxo com o protocolo de seguimento do recém-nascido com sífilis congênita para consulta rápida e de qualidade, caso o profissional de saúde tenha alguma dúvida durante o atendimento do paciente.

O programa possui página de cadastro de pacientes, com dados clínicos referentes ao nascimento (data, sexo, peso e estatura), ao diagnóstico (sífilis congênita sintomática ou assintomática; com ou sem neurossífilis), ao tratamento da sífilis congênita realizado (penicilina benzatina ou procaína ou cristalina) e dados referente aos resultados dos exames (Veneral Disease Research Laboratory-VDRL, punção lombar, hemograma e raio-X de ossos longos) realizados no âmbito hospitalar.

$\mathrm{Na}$ segunda etapa, foram selecionados profissionais médicos pela experiência com diagnóstico e tratamento da patologia para colaborar na validação do conteúdo do instrumento, obedecendo a um número ímpar, a fim de evitar empates nas opiniões durante o processo de validação. Para tanto, utilizou-se uma amostra intencional dos docentes médicos vinculados à Coordenação do curso de Medicina de um Centro Universitário.

Utilizou-se uma busca avançada pelos especialistas, considerando os seguintes critérios de inclusão: ser doutor; pesquisador ou profissional da área da saúde; trabalhar com uma das seguintes temáticas: atenção básica, infectologia e/ou pediatria, conforme preconizado no sistema de pontuação de especialistas em estudos de validação; possuir currículo atualizado nos últimos 60 meses; e que tenha publicado trabalhos ou artigos sobre a temática nos últimos 5 anos. Foram excluídos os especialistas com atuação em outra área, os que se encontravam de férias ou de licença, e os que não responderem dentro dos prazos determinados pelo cronograma desse estudo.

Posteriormente, estabeleceu-se contato com os especialistas selecionados por endereço eletrônico por meio de convite formal explicando a proposta do estudo e, mediante aceite, foi agendada uma data e horário disponível a fim de realizar a avaliação para o processo de validação do programa eletrônico.

Nesta pesquisa, sete especialistas aceitaram participar da pesquisa, constituindo a amostra do estudo. Os dados foram coletados no período de junho a agosto de 2018, por meio de um instrumento de caracterização dos especialistas, com dados referentes à formação e experiência profissional, além de um instrumento de avaliação do protocolo eletrônico constituído por perguntas objetivas a respeito das informações contidas no programa eletrônico quanto aos 'objetivos', 'estrutura e apresentação' e 'relevância', acrescido de espaço para comentários e sugestões, adaptado de questionário preexistente ${ }^{(16)}$ ', com as devidas modificações em relação à temática relacionada ao programa eletrônico deste estudo.

Utilizou-se no instrumento de avaliação uma escala do tipo Likert com pontuação 
de 1 a 4, com as seguintes correspondências: 1- Inadequado, 2 - Parcialmente Adequado; 3 - Adequado, 4 - Totalmente Adequado. Os itens que receberam as pontuações "3" ou "4" foram considerados válidos e os itens pontuados com "1" ou "2" foram eliminados ou revisados.

Conforme preconizado pela literatura, para que a taxa de concordância entre os especialistas fosse considerada aceitável, o IVC deve ser igual ou maior a 0,78. Para o cálculo do IVC por item, somou-se o número de respostas "3" ou "4" e dividiu-se pelo número total de respostas. O IVC médio de cada bloco (Objetivos, Estrutura e Apresentação, e Relevância) foi calculado baseado na média aritmética dos IVC de cada item ${ }^{(17)}$.

Para a obtenção do IVC global do programa eletrônico, foi utilizado o cálculo da média dos valores dos itens calculados, por meio da soma de todos os IVC calculados separadamente, dividido pelo número de itens considerados na avaliação. Os resultados foram apresentados em tabelas a partir dos blocos ${ }^{(17)}$.

O estudo recebeu aprovação do Comitê de Ética em Pesquisa, sob o parecer $\mathrm{n}^{\circ}$ 2.628.947, em 30 de abril de 2018 .

\section{RESULTADOS}

Destacou-se que participaram deste estudo quatro $(57,1 \%)$ especialistas e três $(49,2 \%)$ mestres, todos com experiência em docência e atuação prática na área de interesse e na área de educação em saúde. Foi evidenciado que três $(42,9 \%)$ especialistas possuíam pesquisas publicadas com a temática de sífilis congênita e dois $(28,6 \%)$ com experiência anterior com validação de materiais educativos, quatro $(57,2 \%)$ apresentaram orientações na área de interesse (Tabela 1).

Tabela 1 - Caracterização dos especialistas conforme idade, tempo de formação, titulação, produção científica e experiência profissional nas áreas de interesse. Teresina, PI, Brasil, 2018 (continua)

\begin{tabular}{lll} 
Variáveis & $\mathbf{N}$ & $\%$ \\
\hline Idade & 4 & 57,2 \\
\hline $30-50$ anos & 3 & 42,8 \\
\hline$>50$ anos & & \\
\hline Tempo de formação & 7 & 100 \\
\hline$>16$ anos & 4 & 57,2 \\
\hline Titulação & 3 & 42,8 \\
\hline Especialista & & \\
\hline Mestrado & 3 & 42,8 \\
\hline Pesquisas publicadas em temas relacionados & 2 & 28,6 \\
\hline Sífilis Congênita & 2 & 28,6 \\
\hline Validação de tecnologias educativas & & \\
\hline Outras temáticas & 7 & 100 \\
\hline Experiência como docente e atuação prática em áreas na área de interesse & 3 \\
\hline Sim & 3 & \\
\hline
\end{tabular}


Experiência anterior com validação de materiais educativos

\begin{tabular}{lll}
\hline Sim & 2 & 28,6 \\
\hline Não & 5 & 71,4 \\
\hline
\end{tabular}

Experiência na área da educação em saúde

Sim
7

Orientação de tese/dissertação/monografia na área de interesse

\begin{tabular}{lll}
\hline Sim & 4 & 57,2 \\
\hline Não & 3 & 42,8 \\
\hline Tese ou Dissertação na área de interesse & & \\
\hline Sim & 1 & 14,3 \\
\hline Não & 6 & 85,7
\end{tabular}

No bloco "Objetivos", s especialistas avaliaram os itens sobre coerência com as necessidades profissionais e das atitudes, promoção da mudança de comportamento e atitudes e a possibilidade de circulação no meio científico. Como os três itens deste bloco obtiveram IVC igual a 1,0, o IVC médio também foi igual a 1,0 (Tabela 2).

Tabela 2 - Índice de Validade de Conteúdo (IVC) obtidos para os itens do bloco "Objetivos" do programa eletrônico, conforme a avaliação dos especialistas. Teresina, PI, Brasil, 2018

\section{Objetivos}

São coerentes com as necessidades dos médicos e das atitudes que estes devem ter no seguimento do recém-nascido com sífilis congênita

\begin{tabular}{lllll}
\hline $\begin{array}{l}\text { Promove mudança de comportamento e } \\
\text { atitudes }\end{array}$ & 0 & 0 & 0 & 7 \\
\hline $\begin{array}{l}\text { Pode circular no meio científico na área do } \\
\text { seguimento do recém-nascido com sífilis } \\
\text { congênita }\end{array}$ & 0 & 0 & 0 & 7 \\
\hline
\end{tabular}

IVC médio

Inadequado Parcialmente adequado

Adequado Totalmente IVC Adequado

$\begin{array}{lllll}0 & 0 & 0 & 7 & 1,0\end{array}$


Tabela 3 - Índice de Validade de Conteúdo (IVC) obtidos para os itens do bloco "Estrutura e Apresentação" do programa eletrônico, conforme a avaliação dos especialistas. Teresina, PI, Brasil, 2018

\section{Estrutura e Apresentação}

O programa eletrônico é apropriado para o atendimento e orientação do seguimento do recém-nascido com sífilis congênita
Inadequado Parcialmente Adequado
Adequado Totalmente IVC Adequado

As informações apresentadas estão cientificamente corretas

Há uma sequência lógica do conteúdo proposto

O programa está adequado ao nível sociocultural do público-alvo proposto

As informações encontram-se bem 0

0 6 1 estruturadas em concordância e ortografia

A redação corresponde ao nível de conhecimento do público-alvo

As informações sobre a apresentação do programa encontram-se coerentes

\begin{tabular}{llllll}
\hline As ilustrações são expressivas e suficientes & 0 & 1 & 0 & 6 & 0,86 \\
\hline $\begin{array}{l}\text { O tamanho (extensão do programa) parece } \\
\text { adequado }\end{array}$ & 0 & 1 & 0 & 6 & 0,86 \\
\hline $\begin{array}{l}\text { O título e os tópicos apresentados estão } \\
\text { adequados }\end{array}$ & 0 & 1 & 0 & 6 & 0,86 \\
\hline IVC médio & & & 0,94
\end{tabular}

A Tabela 4 apresenta as respostas obtidas pelos especialistas e o IVC de cada item do terceiro bloco de avaliação, composto por quatro itens, avaliando-se o grau de significação (relevância) do programa eletrônico. Dos quatro itens avaliados, três obtiveram IVC máximo $(1,0)$ e apenas um obteve o IVC $=0,86$. O IVC médio do bloco foi 0,96 .

Tabela 4 - Índice de Validade de Conteúdo (IVC) obtidos para os itens do bloco "Relevância" do programa eletrônico, conforme a avaliação dos especialistas. Teresina, PI, Brasil, 2018 (continua)

\begin{tabular}{|c|c|c|c|c|c|}
\hline Relevância & Inadequado & $\begin{array}{c}\text { Parcialmente } \\
\text { Adequado }\end{array}$ & Adequado & $\begin{array}{l}\text { Totalmente } \\
\text { Adequado }\end{array}$ & IVC \\
\hline $\begin{array}{l}\text { Os temas retratam os aspectos chaves que } \\
\text { devem ser reforçados }\end{array}$ & 0 & 0 & 0 & 7 & 1 \\
\hline $\begin{array}{l}\text { O programa propõe aos profissionais da } \\
\text { atenção básica adquirir conhecimento que } \\
\text { poderá auxiliar no seguimento do recém- } \\
\text { nascido com sífilis congênita }\end{array}$ & 0 & 0 & 0 & 7 & 1 \\
\hline $\begin{array}{l}\text { O programa aborda assuntos necessários } \\
\text { para a prevenção e enfretamento da sífilis } \\
\text { congênita }\end{array}$ & 0 & 1 & 1 & 5 & 0,86 \\
\hline
\end{tabular}


O IVC global do programa eletrônico foi de 0,97 , o que ratificou a validação da aparência e conteúdo. Após reflexão sobre as sugestões dos especialistas, o programador foi contatado a fim de desenvolver alterações nas figuras e textos, e foi obtida, assim, a versão final do programa eletrônico validada.

Na Figura 1, encontram-se representadas as interfaces do programa eletrônico: o cadastro dos dados pessoais dos pacientes, registro dos resultados dos exames dos pacientes para acompanhamento sistemático e base para as informações do seguimento clínico do recém-nascido com sífilis congênita conforme o protocolo do Ministério da Saúde, dados das consultas subsequentes realizadas, gráficos que comparam e representam as variações do VDRL, do peso, estatura e o perfil clínico dos pacientes atendidos na unidade de saúde.
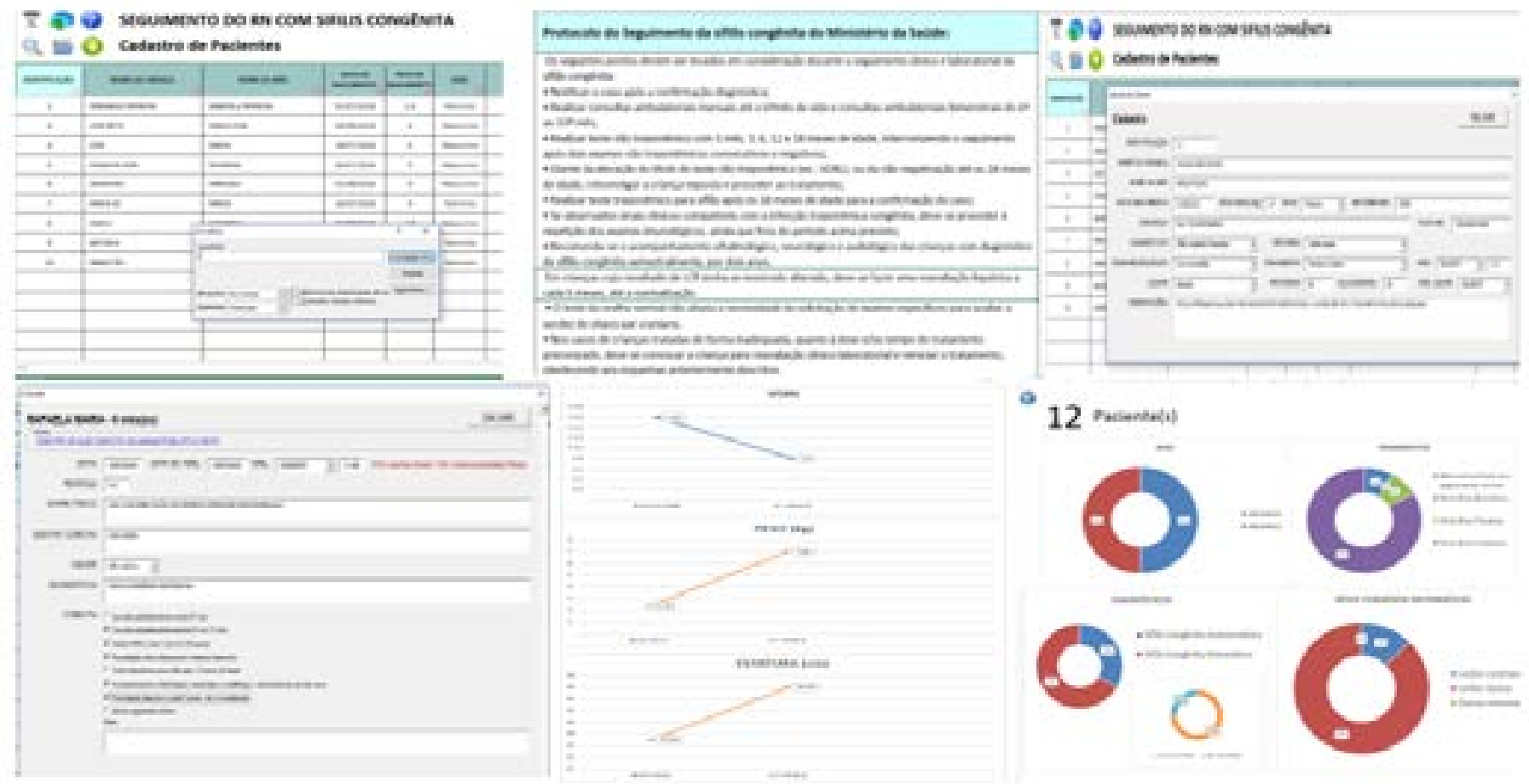

Figura 1 - Versão final do programa eletrônico do seguimento do recém-nascido com sífilis congênita. Teresina, PI, Brasil, 2018

Neste estudo, o programa eletrônico "Seguimento do recém-nascido com sífilis 
congênita" foi validado por especialistas médicos infectologistas ou pediatras, com experiência na área de validação de tecnologias educativas e/ou em sífilis congênita.

Na etapa Índice de Validade de Conteúdo e avaliação dos especialistas sobre os objetivos a serem atingidos com a utilização do programa eletrônico, obteve-se taxa de concordância máxima para todos dos itens.

Já na etapa acerca da estrutura e apresentação do programa eletrônico, na união do consenso entre os especialistas, verificou-se a necessidade de revisão de quatro itens por apresentarem-se como parcialmente adequados. A fim de assegurar a coerência e a coesão da ferramenta, a capa, contracapa, agradecimentos e/ou apresentação foram simplificados para permitir o melhor entendimento, as ilustrações ficaram mais expressivas e o número de páginas e o tamanho do título foram diminuídos.

Desta forma, durante a fase de validação do grau de significação do programa eletrônico, foi possível realizar alterações que abordassem os assuntos necessários para a prevenção e enfretamento da sífilis congênita. Os especialistas afirmaram compreender o programa, porém houve sugestões para acrescentar informações desta temática.

Esta tecnologia representa uma inovação tecnológica, tendo em vista que, embora o tema sífilis congênita e aspectos preventivos na atenção básica sejam discutidos na literatura científica, foram encontradas poucas tecnologias validadas que abordassem a temática, principalmente destinada aos enfermeiros e profissionais de saúde da atenção básica.

Ressalta-se que sífilis congênita apresenta-se como um grande problema de saúde pública e demanda que os serviços de saúde estejam preparados para fazer o seguimento destes pacientes por profissionais de saúde durante todo o período de follow-up ${ }^{(18)}$. A validação de programas eletrônicos é considerada relevante para o planejamento em saúde, tendo em vista que pode apontar para a necessidade de introdução de medidas de cuidado e preventivas ${ }^{(19-20)}$.

No acompanhamento da sífilis congênita, ocorrem frequentes irregularidades, principalmente nos registros, a ausência dos resultados de exames e carência de informações no prontuário, ficando evidente o despreparo e falta de infraestrutura dos profissionais de saúde nos serviços de assistência ${ }^{(21-22)}$.

A validação de um programa eletrônico para seguimento clínico do recém-nascido com sífilis pode colaborar na qualificação dos registros, em todos os níveis de assistência, ao ponto de reconhecermos as situações de risco e a provisão de cuidados para a sobrevida das crianças recém-nascidas com sífilis ${ }^{(23-24)}$.

Diante das estatísticas crescentes da sífilis congênita no Brasil, da insegurança dos profissionais de saúde sobre uma sistematização do acompanhamento ambulatorial da patologia e da possibilidade do surgimento da doença na criança, torna-se necessário padronizar o seguimento desta doença ${ }^{(25)}$.

Essa ferreamente é vantajosa para os enfermeiros e profissionais de saúde, considerando que se trata de um programa que contempla as dimensões: manejo clínico inicial, seguimento terapêutico, parâmetros dos serviços de saúde e conhecimento da doença, e dados que possibilitam estatísticas para o planejamento de ações de prevenção. Quando comparado ao prontuário eletrônico, esse programa eletrônico possui o benefício de ser uma tecnologia móvel de baixo custo com potencial de abrangência geográfica.

Com o auxílio de um programa eletrônico para realização da consulta, o profissional de saúde poderá ter o protocolo de seguimento dos pacientes de forma acessível e informatizada, o que possibilita o envio de relatórios semestrais à Secretaria de Saúde ${ }^{(26)}$.

Além disso, outras ferramentas informatizadas, como os aplicativos móveis, podem notificar a adesão ao tratamento por meio do comparecimento das mães/crianças às 
consultas previamente agendadas, realização de exames complementares, avaliações de outras especialidades e tratamento complementar com os demais profissionais de saúde, tais como fisioterapeutas e fonoaudiólogos ${ }^{(27)}$.

Neste contexto, o desenvolvimento desse programa eletrônico permite também - acompanhamento dos parâmetros clínicos para a compreensão das dimensões do tratamento e facilidade do manejo desses pacientes à medida que permitirá a detecção do cumprimento dos requisitos de autocuidado no pós-parto e puericultura.

A participação dos especialistas no processo de validação da tecnologia educativa promoveu o aperfeiçoamento do programa até a obtenção da versão final, o que a tornou válida de aparência e conteúdo no meio científico. A avaliação dos especialistas configurase por uma rede de profissionais com proficiência e competência em uma esfera específica cujo conhecimento se mostra relevante ${ }^{(28)}$.

Durante o processo de validação, foi possível realizar alterações que respeitassem as particularidades da população-alvo do programa eletrônico com o intuito de que a versão final seja bem compreendida, para ser facilmente utilizada pelos profissionais de saúde da atenção básica.

A respeito das limitações deste estudo, possivelmente, uma delas é a predominância de especialistas médicos, da região Nordeste do Brasil, o que pode sugerir tendência aos hábitos locais e regionais. Dessa forma, a fim de reduzir essa limitação, sugere-se como estudo futuro uma avaliação do programa eletrônico por outros profissionais da saúde de outras regiões brasileiras.

Além disso, destaca-se que, embora o número de estudos científicos relacionados à validação de tecnologias educativas na saúde venha ocupando maiores proporções, ainda há carência de publicações sobre validação de tecnologias que possibilitem auxiliar os profissionais de saúde em ações de seguimento, tratamento e educação em saúde, principalmente na área temática desse estudo, para uma fundamentação mais aprofundada nas análises desta pesquisa ${ }^{(29-30)}$.

Como estudos futuros, sugere-se a validação do programa eletrônico pelo públicoalvo, visando aprimorar esta tecnologia elaborada e validada pelos especialistas. Após a validação, o link ou um QR Code pode ser disponibilizado aos profissionais para que possam acessar o programa.

\section{CONCLUSÃO}

O programa eletrônico proposto foi validado quanto ao conteúdo e aparência por especialistas com experiência em Atenção Básica e/ou sífilis congênita, com IVC global de 0,97 , tendo como contribuição, aos enfermeiros e médicos da Atenção Básica, a produção de uma ferramenta de fácil utilização para o efetivo seguimento clínico ao recém-nascido com sífilis congênita.

No processo de adaptação do programa eletrônico, as sugestões dos especialistas foram primordiais para tornar a tecnologia com maior rigor científico e maior facilidade de utilização para uma adequada sistematização do seguimento clínico da doença pelos profissionais de saúde.

Elaborar e validar esse programa eletrônico contribui para a prática clínica e científica, pois representa uma inovação para a tomada de decisão prática no seguimento do RN com sífilis congênita, além de contribuir para sociedade na obtenção de melhores resultados no cuidado a esse público.

Dessa forma, o resultado deste estudo poderá possibilitar uma reflexão sobre a 
necessidade de ampliar este programa ao pré-natal e parto, além do seguimento pósnatal, a fim de contemplar todos os estágios da vida da criança.

\section{REFERÊNCIAS}

1. Andrade ALMB, Magalhães PVVS, Moraes MM, Tresoldi AT, Pereira RM. Diagnóstico tardio de sífilis congênita: uma realidade na atenção à saúde da mulher e da criança no brasil. Rev. paul. pediatr. [Internet]. 2018 [acesso em 15 out 2018]; 36(3). Disponível em: http://dx.doi.org/10.1590/19840462/:2018;36;3;00011.

2. Bellucci Júnior JA, Vituri DW, Versa GLG da S, Furuya PS, Vidor RC, Matsuda LMA. Acolhimento com classificação de risco em serviço hospitalar de emergência: avaliação do processo de atendimento. Rev. enferm. ỦERJ. [Internet]. 2015 [acesso em 11 jul 2018]; 23(1). Disponível em: http://dx.doi.org/10.12957/ reuerj.2015.4976.

3. Vallejo $C$, Cifuentes Y. Caracterización y seguimiento durante seis meses de una cohorte de recién nacidos con sífilis congénita. Biomedica [Internet]. 2016 [acesso em 6 jun 2018]; 36(1). Disponível em: https://doi.org/10.7705/biomedica.v36i1.2661.

4. Tsimis ME, Sheffield JS. Update on syphilis and pregnancy. Birth Defects Res A Clin Mol Teratol [Internet]. 2017 [acesso em 4 out 2018]; 109(5). Disponível em: https://doi.org/10.1002/bdra.23562.

5. Ministério da Saúde (BR). Departamento de Vigilância, Prevenção e Controle das IST, do HIV/Aids e das Hepatites Virais. Boletim Epidemiológico Sífilis. Brasília: Ministério da Saúde; 2017.

6. Sonda EC, Richter FF, Boschetti G, Casasola MP, Krumel CF, Machado CPH. Congenital Syphilis: literature review. Rev Epidemiol Control Infect [Internet]. 2013 [acesso em 11 jul 2018];3(1). Disponível em: https://doi.org/10.17058/reci.v3i1.3022.

7. Brignol S, Dourado I, Amorim LD, Kerr LRFS. Vulnerability in the context of HIV and syphilis infection in a population of men who have sex with men (MSM) in Salvador, Bahia State, Brazil. Cad. Saúde Pública [Internet]. 2015 [acesso em 10 mar 2018]; 31(5). Disponível em: http://dx.doi.org/10.1590/0102$311 \times 00178313$.

8. Cooper JM, Michelow IC, Wozniak PS, Sánchez PJ. In time: the persistence of congenital syphilis in Brazil - More progress needed! Rev. paul. pediatr. [Internet]. 2016 [acesso em 9 out 2018]; 34(3). Disponível em: http://dx.doi.org/10.1016/j.rppede.2016.06.004.

9. World Health Organization (WHO). Global guidence on criteria and processes for validation: elimination of mother-to-child transmission of HIV and syphilis [Internet]. Geneva: World Health Organization; 2017 [acesso em 10 set 2018]. Disponível em: http://apps.who.int/iris/bitstream/handle/10665/259517/9789241513272-eng. pdf;jsessionid=EF837D9D9AD0FC4D9C67A55DC448E490? sequence=1.

10. Soares LG, Zarpellon B, Soares LG, Baratieri T, Lentsck MH, Mazza V de A. Gestational and congenital syphilis: maternal, neonatal characteristics and outcome of cases. Rev. Bras. Saude Mater. Infant. [Internet]. 2017 [acesso em 28 set 2018]; 17(4). Disponível em: http://dx.doi.org/10.1590/180693042017000400010.

11. Cardoso ARP, Araújo MAL, Cavalcante MS, Frota MA, Melo SP de. Analysis of cases of gestational and congenital syphilis between 2008 and 2010 in Fortaleza, State of Ceará, Brazil. Cienc. saude colet. [Internet]. 2018 [acesso em 9 out 2018]; 23(2). Disponível em: http://dx.doi.org/10.1590/1413$\underline{81232018232.01772016 .}$

12. Pegoraro LG de O, Gvozd R, Haddad M do CFL, Vannuchi MTO, Silva LG de C, Rossaneis MA. Validation of instrument to assess software of patients' risk classification. Rev. Bras. Enferm. [Internet]. 2018 [acesso em 25 out 2018]; 71(3). Disponível em: http://dx.doi.org/10.1590/0034-7167-2017-0053. 
13. Echer IC. Elaboração de manuais de orientação para o cuidado em saúde. Rev. Latino-Am. Enfermagem [Internet]. 2005 [acesso em 02 set 2019]; 13(5):754-7. Disponível em: http://dx.doi. org/10.1590/S0104-11692005000500022.

14. Polit DF, Beck CT, Hungler BP. Fundamentos de pesquisa em enfermagem: métodos, avaliação e utilização. Porto Alegre: Artmed; 2011.

15. Melo RP, Moreira RP, Fontenele FC, Aguiar ASC de, Joventino ES, Carvalho EC de. Criteria for selection of experts for validation studies of nursing phenomena. Rev. RENE. [Internet]. 2011 [acesso em 3 nov 2018]; 12(2). Disponível em: http://periodicos.ufc.br/rene/article/view/4254/3285.

16. Oliveira MS de, Fernandes AFC, Sawada NO. Manual educativo para o autocuidado da mulher mastectomizada: um estudo de validação. Texto contexto-enferm.[Internet]. 2008 [acesso em 11 set 2018]; 17(1). Disponível em: http://dx.doi.org/10.1590/S0104-07072008000100013.

17. Alexandre NMC, Coluci MZO. Content validity in the development and adaptation processes of measurement instruments. Cienc. saude colet [Internet]. 2011 [acesso em 9 nov 2018]; 16(7). Disponível em: http://dx.doi.org/10.1590/S1413-81232011000800006.

18. Padovani C, Oliveira RR de, Pelloso SM. Syphilis in during pregnancy: association of maternal and perinatal characteristics in a region of southern Brazil. Rev. Latino-Am. Enfermagem [Internet]. 2018 [acesso em 10 nov 2018]; 26. Disponível em: http://dx.doi.org/10.1590/1518-8345.2305.3019.

19. Silva BS, Coelho HV, Cavalcante RB, Oliveira VC de, Guimarães EA de A. Evaluation study of the National Immunization Program Information System. Rev. bras. enferm. [Internet]. 2018 [acesso em 5 nov 2018]; 71(1). Disponível em: http://dx.doi.org/10.1590/0034-7167-2017-0601.

20. Carreno I, Moreschi C, Marina B, Hendges DJB, Rempel C, Oliveira MMC de. Analysis of the use of data from the Primary Health Care Information System (SIAB): an integrative review of the literature. Cienc. saude colet. [Internet]. 2015 [acesso em 10 set 2018]; 20(3). Disponível em: http://dx.doi. org/10.1590/1413-81232015203.17002013.

21. Lazarini FM, Barbosa DA. Intervenção educacional na atenção básica para prevenção da sífilis congênita. Rev. Latino-Am. Enfermagem [Internet]. 2017 [acesso em 21 set 2018]; 25. Disponível em: http://dx.doi.org/10.1590/1518-8345.1612.2845.

22. Kawaguchi IAL, Magalhães DM dos S, Calderon I de MP, Dias A. O seguimento da sífilis congênita em crianças tratadas ao nascer. Com. ciênc. saúde [Internet]. 2014 [acesso em 02 set 2018]; 24(3). Disponível em: http://hdl.handle.net/11449/136933.

23. Alpirez LA, Lopes Neto D, Moisés MS, Dias VP. Content validation of an infant evaluation instrument. Acta Paul Enferm.[Internet]. 2018 [acesso em 01 set 2019]; 31(2):123-9. Disponível em: http://dx.doi. org/10.1590/1982-0194201800019.

24. Pinilla G, Campos L, Durán A, Navarrete J, Muñoz L. Detección de treponema pallidum subespecie pallidum para el diagnóstico de sífilis congénita mediante reacción en cadena de la polimerasa anidada. Biomedica [Internet]. 2018 [acesso em 01 nov 2019]; 38(1):128-35. Disponível em: http://doi.org/10.7705/ biomedica.v38i0.3740.

25. Macêdo VC de, Lira PIC de, Frias PG de, Romaguera LMD, Caires SFF, Ximenes RAA. Fatores de risco para sífilis em mulheres: estudo caso-controle. Rev. Saúde Pública [Internet]. 2017 [acesso em 5 nov 2018]; 51. Disponível em: https://doi.org/10.11606/s1518-8787.2017051007066.

26. Pereira IM, Bonfim D, Peres HHC, Goes RF, Gaidzinski RR. Mobile application for data collection in health research. Acta Paul. Enferm. [Internet]. 2017 [acesso em 2 nov 2018]; 30(5). Disponível em: http:// dx.doi.org/10.1590/1982-0194201700069.

27. Rocha TAH, Fachini LA, Thumé E, Silva NC da, Barbosa ACQ, Carmo M do, Rodrigues JM. Saúde Móvel: novas perspectivas para a oferta de serviços em saúde. Epidemiol. Serv. Saúde [Internet]. 2016 [acesso em 11 out 2018]; 25(1). Disponível em: http://www.scielo.br/scielo.php?pid=S2237$96222016000100159 \&$ script $=$ sci abstract. 
28. Lago RF do, Costa N do R. Expert communities and interest-formation in the Brazilian AIDS program. Cienc. saude colet. [Internet]. 2017 [acesso em 23 out 2018];22(5). Disponível em: http://dx.doi. org/10.1590/1413-81232017225.33512016.

29. Wedderburn CJ, Murtagh M, Toskin I, Descascamento RW. Using electronic readers to monitor progress toward elimination of mother-to-child transmission of HIV and syphilis: an opinion piece. Int J Gynaecol Obstet [Internet]. 2015 [acesso em 01 nov 2019]; 130(Suppl 1). Disponível em: http://dx.doi. org/10.1016/j.ijgo.2015.04.006.

30. Yi J, Choi W, Shin S, Choi J, Kim H, Chung HJ, et al. Strategy for performing treponemal tests in reverse-sequence algorithms of syphilis diagnosis. Clin Biochem [Internet]. 2019 [acesso em 01 nov 2019]; 63. Disponível em: http://dx.doi.org/10.1016/j.clinbiochem.2018.09.013.

Recebido: 14/11/2018

Finalizado: 04/03/2020

Autor Correspondente:

Camila Aparecida Pinheiro Landim Almeida

Centro Universitário Uninovafapi

R. Vitorino Orthiges Fernandes, 6123 - 64073-505 - Teresina, PI, Brasil

E-mail: camila@uninovafapi.edu.br

Contribuição dos autores:

Contribuições substanciais para a concepção ou desenho do estudo; ou a aquisição, análise ou interpretação de dados do estudo - JCSS, CVR

Elaboração e revisão crítica do conteúdo intelectual do estudo - ETHA

Aprovação da versão final do estudo a ser publicado - LKBM

Responsável por todos os aspectos do estudo, assegurando as questões de precisão ou integridade de qualquer parte do estudo - SFVV, CAPLA 